\title{
THE ELECTRODYNAMICS OF NEUTRINOS IN DISPERSIVE MEDIA
}

\author{
V.N. Oraevsky, V.B. Semikoz
}

1. Neutrinos interacting with the vacuum of vector bosons and leptons possess important vacuum electromagnetic characteristics: viz., the anomalous magnetic moment $\Delta \mu_{v}^{\mathrm{vac}}$ and the mean-square radius of charge distribution $\left\langle\mathrm{r}^{2}>1 / 2\right.$. As a result, neutrinos, just like neutrons, may interact with an external magnetic field.

The magnitude of any electromagnetic characteristic of a neutrino is proportional to the Fermi weak interaction constant $\mathrm{G}_{\mathrm{F}}=10^{-5} / \mathrm{m}_{\rho}^{2} *$ ), so that in a vacuum electromagnetic interactions determine only radiation corrections $(\mathrm{RC})$ to the principal Born contribution to the cross sections of weak processes.

However, in a dispersive medium (DM), say in a plasma, the electromagnetic interaction of a neutrino with charged particles and the external magnetic field is much more pronounced. Furthermore, in the low-energy range, where the neutrino de Broglie wavelength $\lambda_{v}=\mathrm{p}^{-1}$ is greater than or equal to the mean distance between particles, $\mathrm{n}_{0}^{-1 / 3}$, the contribution of electromagnetic processes with the neutrinos involved may be higher than the contribution due to ordinary weak interactions, i.e. the electromagnetic contribution can no longer be considered a small correction, as in the case of RC in a vacuum.

2. In the case of DM, a neutrino acquires new characteristics; an induced electric charge [1,2] and a magnetic moment [3], depending on the frequency spectrum and on the polarization of the external field, and also on the polarization of electromagnetic radiation of the neutrino itself.

Special emphasis is given to the polarization origin of electric charge, due to an inhomogeneous electron concentration around a neutrino.

The magnitude of the induced electric charge

* Here we use the system of units in which $\mathrm{h}=\mathrm{c}=1$.

E. R. Priest and V. Krishan (eds.), Basic Plasma Processes on the Sun, 35-38.

(C) 1990 IAU. Printed in the Netherlands. 


$$
e_{v}^{\text {ind }}=\frac{G_{F}(1+4 \xi) e}{4 \pi d \sqrt{2} r_{D}^{2}}
$$

per electron charge $e_{v}^{\text {ind }} / e$ ) is equal approximately to $10^{-16}$ for metal or the Sun, and $10^{-8}$ for a collapsing star. Here $\xi=\sin ^{2} \theta_{w}$ is the parameter of Weinberg-Salam's model ( $\xi$ 0.23 from experiment); $\alpha=e^{2}=137^{-1} ; r_{D}$ is the Debye radius of the plasma.

The concept of neutrino "electric charge" is illustrated by considering the electromagnetic part of the total amplitude of elastic neutrino scattering from nuclei in a plasma. It is shown that, if the contribution due to neutral currents is neglected, the situation is completely analogous to Mott's scattering of an electron from a Coulomb centre in the same medium [4].

We obtain the total electromagnetic vortex of a neutrino in an isotropic medium, which determines the corresponding electromagnetic current [5]. The expression for the electromagnetic current is used to derive lower-order multipole moments of a neutrino in an isotropic medium [6]. We consider also a specific neutrino electromagnetic moment (Zeldovich anapole) caused by the non-conservation of parity [7].

3. The many-particle equations of motion of neutrinos and electrons - the relativistic kinetic equations (RKE) - are derived in [8]. The most important feature of electrodynamics of neutrinos in a DM is the presence in the RKE of the contribution due to the self-consistent electromagnetic field, which has never been taken into account in the conventional treatment of relativistic neutrino kinetics. The RKE for neutrinos and electrons, obtained by the Bogolyubov method using Feynman diagrams for the vesystem, contain macroscopic effects of spatial parity violation due to pseudovector currents in the scattering. We derive also the integral of ve collisions under dynamic polarization of the medium. This integral (of the Lenard-Balesku type) is a typical integral occurring in plasma electrodynamics, and is of great importance for applications in the theory of collapsing stars.

4. The applications concerning elementary processes in a DM with neutrinos are considered. The cross-section of elastic neutrino scattering from spinless nuclei under the collapse of a presupernova star is calculated [4]. It is shown that a change in the electromagnetic structure of electron neutrinos in a DM reduces (screens) the cross section of VA scattering by approximately a factor of 10 as compared with the standard Born cross section calculated in the standard model for the collapse conditions. As for $v_{\mu}$-neutrino, the cross section of $v A$-scattering changes slightly in comparison with the standard Born cross section. Therefore, in a dense plasma the diffusion of neutrinos of distinct flavours occurs differently.

5. In the symmetric model of electroweak interactions with right currents we predict a change of neutrino helicity in a plasma (left neutrinos $\leftrightarrow$ right neutrinos) without including the vacuum magnetic moment $\Delta \mu_{v}^{\mathrm{vac}}$ of the neutrino [9]. 
A change of neutrino helicity in a supernova star results in a reduction of the observed left neutrino flux and in a restriction for the mixing angle of a left- and right Wboson ( $\sin 2 \zeta \leq 0.4 \mathrm{E} / \mathrm{pF}$ ) which is the important parameter of a symmetric model. Here $\mathrm{E}$ is the neutrino energy, $\mathrm{pF}_{\mathrm{F}}$ is the Fermi momentum of electrons with density $\mathrm{n}_{0}=$ $\mathrm{p}_{\mathrm{F}}^{3} / 3 \pi^{2}$. In the symmetric mode a neutrino has two helicity states due to the existence of an effective neutrino rest mass [10]:

$$
\mathrm{m}_{\mathrm{eff}}^{\text {eff }}=\frac{\mathrm{G}_{\mathrm{F}}\left(\varepsilon^{2}-1\right)}{\sqrt{2}} \mathrm{~m}_{\mathrm{e}} \int \frac{\mathrm{d}^{3} \mathrm{p}}{\varepsilon_{\mathrm{p}}}\left[\mathrm{f}_{0}^{(\mathrm{e})}\left(\varepsilon_{\mathrm{p}}\right)+\underset{0}{\left.\mathrm{f}^{(\widetilde{\mathrm{e}})}\left(\varepsilon_{\mathrm{p}}\right)\right]}\right.
$$

where $\varepsilon=(1+\operatorname{tg} \zeta) /(1-\operatorname{tg} \zeta)$ is the parameter of the same model; $m_{e}$ is the electron rest mass; $\mathrm{f}_{0}^{(\mathrm{e})}\left(\varepsilon_{\mathrm{p}}\right), \mathrm{f}_{0}^{(\widetilde{e})}\left(\varepsilon_{\mathrm{p}}\right)$ are the Fermi distribution functions of electrons and positrons. In a vacuum $\left(f_{0}^{(e)}=f_{0}^{(\widetilde{e})}=0\right)$ or without right currents $(\zeta=0, \varepsilon=1)$ the mass $m_{v}^{\text {eff }}$ is zero.

6. Thus, in a dispersive medium a neutrino acquires electromagnetic properties which resemble, in many respects, the properties of an electron under the same conditions: the electromagnetic interaction of a neutrino with charged particles can be screened at distances of the order of the Debye radius; in a medium a neutrino can emit and absorb electromagnetic waves (Vavilov-Cerenkov radiation and Landau's damping of plasma waves), etc. For an electron which, besides electric charge, possesses vacuum weak charge (both vector and pseudovector) weak interaction with other electrons and nuclei is only a small correction to the electromagnetic interaction in a DM. On the contrary, for neutrinos (under the same conditions) the electromagnetic interaction with electric charges is stronger than the weak interaction caused by the existence of only a bare weak nuetrino charge in vacuum. This distinguishes the behaviour of low-energy neutrinos in a DM from the corresponding behaviour in a vacuum (or in a dielectric) where the electromagnetic interaction is only a $\mathrm{RC}$ as compared with the point Fermi weak interaction.

\section{References}

1. V N Oraevsky and V S Semikoz, Physica 142A, 135 (1987).

2. V N Oraevsky and V B Semikoz, Proceedings of the Joint Varenna-Abastumani International School and Workshop on "Plasma Astrophysics" Sukhumi, USSR, 19-28 May 1986, p.321.

3. V B Semikoz, Sov. J. Nucl. Phys. 46, 946 (1987).

4. L B Leinson, V N Oraevsky and V B Semikoz, Phys. Lett. 209B, 80 (1988).

5. V N Oraevsky, A Yu Plakhov, V B Semikoz and Ya A Smorodinsky, Zh. Eks. Theor. Fiz. 93, 1157 (1987) (Sov. Phys. JETP, 66, 890 (1987)) Errata in Zh. Eks. Theor. Fiz. 95, p.39 (1989); ibid. 95, N6, (1989).

6. V. B Semikoz and Ya A Smorodinsky, Zh. Eks. Theor. Fiz. 96, 35 (1989) (Sov. Phys. JETP, 68, 20 (1989). 
7. V B Semikoz and Ya A Smorodinsky, Pis'ma Zh. Eksp. Theor. Phys. 48, 361 (1988) (Sov. Phys. JETP Lett. 48, 399 (1988)).

8. V B Semikoz, Physica 142A, 157 (1987).

9. V B Semikoz, Pis'ma Zh. Eksp. Theor. Phys. 49, 254 (Sov. Phys. JETP Lett. 49, 288 (1989)).

10. V N Oraevsky, V B Semikoz and Ya A Smorodinsky, Phys. Lett. 227B, 255 (1989).

\section{DISCUSSION}

SIVARAM: (i) How would the presence of a large magnetic field affect your result for helicity flip?

(ii) Can you comment on the limits of the neutrino magnetic moment in these processes?

SEMIKOZ: (i) If a magnetic field is taken into account, the collective interaction of neutrinos with matter (introduced by us) would be more important than a simple inclusion of the vacuum magnetic moment of neutrinos which interacts with the magnetic field too. The helicity flip occurs both due to the vacuum magnetic moment and due to our effective magnetic moment. However the vacuum helicity flip is suppressed by dense matter (which is the main reason why the convective zone is considered) for this effect!), but our channel of helicity flip increases with electron density.

(ii) The laboratory upper limit (Reines et al) is known to be

$$
\mu_{v}^{\mathrm{vac}} \leq 10^{-10} \text { Bohr magneton }\left(\mu_{\mathrm{B}}\right) \text {. }
$$

The astrophysical restriction is stronger. The cooling times of white dwarfs (about $\sim 400$ stars) imply

$$
\mu_{v}^{\mathrm{vac}} \leq 10^{-11} \mu_{\mathrm{B}} \quad \text { (S Blinnikov (USSR), 1988). }
$$

Also the restriction of a possible right (sterile) neutrino flux emitted by SN 1987A, which could be converted into left neutrinos at the Earth (after crossing the Galaxy's magnetic field) gives the limit (D Nötzold, 1988)

$$
\mu_{v}^{\mathrm{vac}} \leq 10^{-12} \mu_{\mathrm{B}}
$$

ORAEVSKY: In a medium an induced charge of the neutrino is created: its value is proportional to the Fermi Constant and the electron density. There is also an induced magnetic moment, its value for the Supernova is $\sim 10^{-10}$ of a Bohr magneton, which is 9 orders of magnitude higher than the vacuum value. In a medium, due to coherent interaction with particles, an induced neutrino mass is created. 